\title{
EDITORIAL
}

Korean J Intern Med 2022;37:283-284

https://doi.org/10.3904/kjim.2022.043

\section{Simple renal cysts: no longer simple?}

\author{
Sang Heon Suh and Eun Hui Bae
}

Department of Internal Medicine, Chonnam National University Medical School, Gwangju, Korea

Received : January 19, 2022

Accepted: January 29, 2022

\section{Correspondence to}

Eun Hui Bae, M.D.

Department of Internal Medicine, Chonnam National University Medical School, 42 Jebong-ro, Dong-gu, Gwangju 61469, Korea Tel: +82-62-220-6503

Fax: +82-62-225-8578

E-mail: baedak76@gmail.com https://orcid.org/0000-0003-17272822

\section{See Article on Page 425-433}

Simple renal cysts are common acquired lesions, and their prevalence increases with age, ranging from $6 \%$ in adults 21 to 40 years of age to $35 \%$ in those $>70$ years of age [1]. The Bosniak Classification of Cystic Renal Masses provides radiologic criteria for simple renal cysts (Bosniak class I): a cystic lesion with a well-defined, thin $(<2 \mathrm{~mm})$, smooth wall and homogeneous simple fluid, and without septa or calcifications as seen on computed tomography or magnetic resonance imaging [2]. An ultrasonographically based definition (i.e., a round or oval cyst with a thin, smooth, sharply marginated wall and no evidence of calcification or malignancy), is also commonly accepted [3]. A simple renal cyst is, by definition, benign and does not require further follow-up unless the patient is symptomatic [2].

Despite the lack of malignant potential, mounting evidence suggests that a simple renal cyst is in fact not simple. A cross-sectional analysis of 872 1:1-matched patients from a single center in Korea showed that the presence of a cyst is clearly associated with hypertension (HTN) and that the number of cysts as well as their size are risk factors for HTN [4]. Another study, based on cross-analyses of 14,995 patients, found that simple renal cysts are associated with prehypertension as well as HTN [5] and that two or more simple renal cysts and cysts $\geq 2 \mathrm{~cm}$ are important determinants of prehypertension and HTN in adults. As HTN is a major risk factor for the development and progression of chronic kidney disease, the association between simple renal cysts and kidney dysfunction has been suggested, although not without debate [6]. The association of simple renal cysts and elevated serum creatinine was previously determined in a cross-sectional analysis of the general population [1], while in an analysis of 561 hospitalized individuals the presence of renal cysts, regardless of their number, was shown to be associated with reduced estimated creatinine clearance [7]. Taken together, the evidence so far strongly indicates an association of simple renal cysts with functional damage to the renal parenchyma. Consequently, whether simple renal cysts are also related to renal events that precede a reduced glomerular filtration rate, such as albuminuria, merits consideration.

In this issue of the Korean Journal of Internal Medicine, Boo et al. [3] report the results of their comprehensive evaluation of the association of simple renal cysts with hematuria and albuminuria in young adults (age $<40$ years). Among 5,838 patients who underwent a comprehensive medical examination and were generally healthy, simple renal cysts were detected in 276 (cyst group). The prevalence of hematuria in the control versus the cyst group did not significantly differ $(4.9 \%$ vs. $6.5 \%, p=0.243)$, whereas the prevalence of albuminuria was significantly higher in the cyst than in the control group $(11.3 \%$ vs. $4.5 \%, p<0.001)$. The 
number of cysts (single vs. multiple) did not affect the prevalence of albuminuria $(12.0 \%$ vs. $7.1 \%, p=0.363)$. Multivariate regression analysis revealed an independent association of simple renal cysts with albuminuria (adjusted odds ratio, 2.307; 95\% confidence interval, 1.512 to $3.519 ; p<0.001$ ). Support for this finding was obtained in a $1: 2$ propensity score matching analysis, in which the variables were age, sex, body mass index, systolic blood pressure, HTN, diabetes mellitus, estimated glomerular filtration rate, history of smoking, and serum uric acid level. Logistic regression analyses of albuminuria in matched cohorts robustly showed that simple renal cysts are associated with albuminuria (adjusted odds ratio, 1.858; 95\% confidence interval, 1.112 to 3.107; $p=0.0182$ ). Thus, the current study by Boo et al. [3] highlights the potential clinical significance of simple renal cysts. Although it is unclear whether a simple renal cyst is a cause or consequence of kidney damage, the study justifies the close monitoring of patients with simple renal cysts, especially adults $<40$ years, for the development of albuminuria. Additional prospective studies investigating the association of renal cysts with albuminuria are warranted.

A limitation of the study of Boo et al. [3] is that most patients were in their late 30s, such that support for the clinical significance of simple renal cysts in individuals $<30$ years of age was less substantial, as also noted by the authors. In addition, because the authors chose to exclude adults $\geq 40$ years age, the clinical impact of simple renal cysts in older patients and in the elderly remains to be determined.

In summary, the study of Boo et al. [3] demonstrates the association of simple renal cysts with albuminuria in young adults and thus the clinical significance of these benign lesions. Additional prospective studies of the casual relation between renal cysts and albuminuria are needed.

\section{Conflict of interest}

Eun Hui Bae is an Associate Editor of the Korean Journal of Internal Medicine. No potential conflict of interest relevant to this article was reported.

\section{REFERENCES}

1. Chang CC, Kuo JY, Chan WL, Chen KK, Chang LS. Prevalence and clinical characteristics of simple renal cyst. J Chin Med Assoc 2007;70:486-491.

2. Silverman SG, Pedrosa I, Ellis JH, et al. Bosniak classification of cystic renal masses, version 2019: an update proposal and needs assessment. Radiology 2019;292:475-488.

3. Boo HJ, Lee JE, Chung SM, et al. The presence of simple renal cysts is associated with an increased risk of albuminuria in young adults. Korean J Intern Med. 2022;37:425-433.

4. Chin HJ, Ro H, Lee HJ, Na KY, Chae DW. The clinical significances of simple renal cyst: Is it related to hypertension or renal dysfunction? Kidney Int 2006;70:1468-1473.

5. Lee CT, Yang YC, Wu JS, et al. Multiple and large simple renal cysts are associated with prehypertension and hypertension. Kidney Int 2013;83:924-930.

6. Lee HS, Kim SG, Kim EJ, et al. The changes of renal function in apparent healthy individuals with a simple renal cyst. Korean J Nephrol 2007;26:554-558.

7. Al-Said J, Brumback MA, Moghazi S, Baumgarten DA, O'Neill WC. Reduced renal function in patients with simple renal cysts. Kidney Int 2004;65:2303-2308. 